\section{La investigación ambiental en el IF Sertão-PE y su contribución al desarrollo sostenible en los territorios semiáridos del Brasil.}

Clecia Simone Goncalves Rosa Pacheco

Instituto Federal do Sertão Pernambucano, Brasil clecia.pacheco@ifsertao-pe.edu.br

\section{Environmental research in the IF Sertão-PE and its contribution to sustainable development in the semi-aird territories of Brazil.}

Recibido: $12 / 12 / 2017$

Aceptado: $20 / 04 / 2018$

Publicado: 29/06/2018

\title{
RESUMEN
}

El Instituto Federal de Educación, Ciencia y Tecnología del Sertão Pernambucano (IF Sertão-PE) tiene la misión de promover el desarrollo regional sostenible, con énfasis en la ciencia y la tecnología, a través de la enseñanza, la investigación, la extensión e innovación, formando personas capaces de transformar la sociedad. Su visión es ser una institución de excelencia en todos los niveles y modalidades de enseñanza, articulados con la investigación y la extensión, comprometida con la transformación social, fundamentada en la ética y la ciudadanía. El propósito de este artículo es presentar algunos reportes de investigaciones desarrolladas y en desarrollo en el IF Sertão-PE y su relevancia para el desarrollo de los territorios semiáridos, buscando una convivencia sostenible entre la naturaleza y la sociedad, así como destacar el modo en que se desarrollan tales investigaciones y las actividades vinculadas a éstas, como forma práctica de ejecución entre la institución y la sociedad local y regional.

PALABRAS CLAVE: misión, medio ambiente, sustentabilidad, visión.

\section{ABSTRACT}

The Federal Institute of Education, Science and Technology of Sertão Pernambucano (IF Sertão-PE) has the mission of promoting sustainable regional development, with emphasis on science and technology, through teaching, research, extension and innovation, forming people capable of transforming society. Its vision is to be an institution of excellence at all levels and teaching modalities, articulated with research and extension, committed to social transformation, based on ethics and citizenship. The purpose of this article is to present some reports of developed and developing research in the IF Sertão-PE and its relevance for the development of semi-arid territories, seeking a sustainable coexistence between nature and society, as well as highlighting the way in which such investigations and activities related to them are developed, as a practical form of execution between the institution and the local and regional society.

KEYWORDS: environment, mission, sustainability, vision.

\section{INTRODUCCIÓN}

La investigación es el resorte propulsor para la formación de los individuos. Los prepara para desarrollar una mirada científica e investigativa a través de la experiencia práctica y hace emerger todo el potencial humano transformador de extrema complejidad que se expresa en la relación existente entre la sociedad y la naturaleza. 
El Instituto Federal de Educación, Ciencia y Tecnología del Sertão Pernambucano (IF Sertão-PE) tiene como misión promover el desarrollo regional sostenible, con énfasis en la ciencia y la tecnología, a través de la enseñanza, la investigación, la extensión e innovación, buscando la capacitación de personas para transformar la sociedad. Su visión es ser una institución de excelencia en todos los niveles y modalidades de enseñanza, articulada con la investigación, la extensión e innovación, comprometida con la transformación social, con base en la ética y la ciudadanía.

Las investigaciones ambientales en el IF Sertão-PE buscan contribuir al desarrollo sostenible de los actores sociales que viven en los territorios semiáridos, buscando formas de mitiga ción y convivencia sostenible. «El Semiárido es un área considerada de gran vulnerabilidad, no sólo climática sino también socioeconómica. Es una región caracterizada por la fuerte insolación (2 800 horas anuales), presentando temperaturas que varían de $23{ }^{\circ} \mathrm{C}$ a $27{ }^{\circ} \mathrm{C}$, con pluviosidad irregular y mal distribuida» (Pacheco, 2014, p. 45).

Siendo así, el presente artículo se propone presentar algunos reportes de investigaciones desarrolladas y en desarrollo en el IF Sertão-PE y su relevancia para el desarrollo de los territorios semiáridos, buscando una convivencia sostenible entre la naturaleza y la sociedad, así como destacar el modo en que se desarrollan tales investigaciones y las actividades vinculadas a éstas, como forma práctica de ejecución entre la institución y la sociedad local y regional.

\section{Recorte de la investigación en el IF Sertão-PE de 2012 a 2017}

Los Institutos Federales de Educación, Ciencia y Tecnología, deben fomentar el diálogo dentro de su territorio, provocar la actitud de reflexión frente al mundo y dialogar con este mundo en una actitud propia de investigación. En la relación con la investigación, el acto de investigar, en el IF Sertão-PE, está anclado en dos principios: el científico, que se consolida en la construcción de la ciencia; y el educativo, que se refiere a la actitud de cuestionamiento ante la realidad (Instituto Federal Del Sertão Pernambucano [IF SertãoPE], 2016, p.12).

El gran desafío de los Institutos Federales en Brasil en el campo de la investigación, es ir más allá del descubrimiento científico, pues, en su compromiso con la humanidad la investigación debe estar presente en todo trayecto de la formación del trabajador, debe representar la conjugación del saber y de cambiar y construirse, en la indisociabilidad entre investigación, enseñanza, extensión e innovación.

Los nuevos conocimientos producidos por las investigaciones se colocan a favor de los procesos locales y regionales desde una perspectiva de reconocimiento y valorización de los mismos a nivel nacional y global, teniendo en cuenta que se desarrollan en una región semiárida única y atípica en el escenario nacional.

Uno de los caminos para desarrollar la investigación dentro del IF Sertão-PE, son los programas de iniciación científica, cuyo objetivo es: despertar la vocación científica y tecnológica y fomentar talentos entre los discursos a través de la participación en proyectos de investigación, proporcionando a los alumnos la oportunidad de aprender técnicas y métodos científicos, de tal modo que se estimule también a los investigadores productivos a involucrar a los estudiantes en las actividades científicas, tecnológicas y artístico-culturales, para ampliar la participación de la institución en el desarrollo de investigaciones y en la generación de tecnologías aplicadas a los territorios semiáridos, extendiendo sus beneficios a la sociedad. 
Los programas institucionales existentes en el IF Sertão-PE se ejecutan en conformidad con las Resoluciones Normativas No. 015 y 017/2004 del Consejo Nacional de Desarrollo Científico y Tecnológico (CNPq), y con las Resoluciones No. 06/2005 y 06/2007 y con las demás normas del IF Sertão-PE. Entre los programas institucionales se pueden mencionar: el Programa Institucional de Becas de Iniciación Científica (PIBIC) para alumnos de graduación; el Programa Institucional de Becas de Iniciación al Desarrollo Tecnológico e Innovación (PIBITI) para alumnos de graduación; el Programa Institucional de Becas de Iniciación Científica (PIBIC Jr.) para alumnos de nivel medio técnico y subsecuente; y el Programa Institucional Voluntario de Iniciación Científica (PIVIC) para alumnos de cualquier modalidad y nivel.

Los alumnos contemplados para desarrollar las competencias y habilidades previstas por los programas tienen a su disposición un profesor o técnico administrativo orientador, maestro o doctor, con experiencia reconocida en investigación, responsable de la formación del becario para la actividad investigativa; también tienen acceso al Portal de Periódicos de la Coordinación de Perfeccionamiento de Personal de Nivel Superior (CAPES), que posibilita fortalecer el conocimiento a través de diversas publicaciones nacionales e internacionales que contribuyen significativamente al perfeccionamiento de la investigación de forma rápida y gratuita; así mismo tienen acceso a los laboratorios, suplementos y campos experimentales para la realización de sus actividades de investigación, y a los medios de divulgación de los productos de las investigaciones como la Jornada de Iniciación Científica y de Extensión (JINCE) y la revista electrónica Semiárido De Visu.

Además de todo esto, la institución dispone del Programa Institucional de Calificación (PIC) con becas para servidores en capacitación (maestría y/o doctorado) y el Programa Pro-Doctorado (PPD) con becas para auxilio alimentación y vivienda, ofrecidas por la Coordinación de Perfeccionamiento de Personal en Brasil (CAPES) para servidores en capacitación.

Los recursos para el fomento de las becas de los programas descritos provienen de la planificación presupuestaria anual del IF Sertão-PE y de cuotas provenientes de órganos de fomento, como del CNPq. Las cuotas de becas están disponibles a los estudiantes de todos los campus, mediante participación en un proceso selectivo propio realizado a través de llamadas en ediciones específicas que se publican en el sitio electrónico de la Institución.

Los resultados de estos programas para la sociedad, son indiscutiblemente relevantes, ya que propicia la formación de ciudadanos con capacidades en investigación para identificar problemas en sus áreas de actuación, así como de ofrecer soluciones a partir de sus actividades investigativas y el conocimiento científico producido en la ejecución del proyecto de investigación por medio de publicaciones en eventos científicos tales como: jornadas, simposios y congresos, y en revistas científicas, productos, medios y servicios elaborados o mejorados en la investigación realizada.

En la referida institución ha crecido la atención que se da a la innovación tecnológica. La política de innovación tecnológica en el marco del IF Sertão-PE es gestionada por el Núcleo de Innovación Tecnológica (NIT), órgano creado a partir de la Resolución No. 23/2010, cuya misión consiste en transformar la realidad local a través de la investigación aplicada, de la extensión tecnológica y el emprendimiento innovador. Tiene como principal objetivo proteger la creación intelectual derivada de las investigaciones académicas, principalmente a través de depósitos de patentes y de la búsqueda de la transferencia de tecnologías a través del licenciamiento.

EI NIT viene cumpliendo con la finalidad de formar masa crítica para trabajar con innovación tecnológica, ofreciendo servicios de gestión de innovación para los investigadores, becarios de los programas de iniciación científica, PIBIC y PIBIC Jr. y, principalmente, a los becarios del PIBITI, siendo ese la base fundamental de sus acciones. 
El PIBITI tiene como objetivo contribuir a la formación de recursos humanos en actividades de investigación, desarrollo tecnológico e innovación, incentivando, implementando y ampliando las acciones de innovación tecnológica. Además del apoyo ofrecido a los becarios de innovación tecnológica, los servidores también tienen acceso a los servicios ofrecidos por el NIT, tales como prospección tecnológica, protección del conocimiento y de producción industrial, hotel de proyectos e incubación de empresas.

La Incubadora de Empresas del Semiárido (ISA), instituida por la Resolución No. 23 de 31 de mayo de 2010, tiene la misión de fomentar y apoyar acciones de base tecnológica y social como forma de promoción del desarrollo sostenible regional. La ISA, se encuentra vinculada al NIT, desarrollando acciones de fomento y fortalecimiento del emprendimiento en el Valle del San Francisco. Son objetivos de la incubadora: identificar a los emprendedores; fomentar el surgimiento de empresas de base tecnológica; fomentar el surgimiento de empresas de base social; aproximar el IF Sertão-PE del sector productivo; propiciar nuevas oportunidades de trabajo por la implementación de empresas de base tecnológica y social; contribuir al desarrollo de los arreglos productivos locales en nuestra región (IF Sertão-PE, 2016).

El IF Sertão-PE también ofrece cursos de postgrado lato sensu, y se prepara para crear programas de postgrado stricto sensu, implantando acciones que favorecen la realización de esos cursos, de forma que tengan su excelencia reconocida.

Hasta el año 2016, este instituto ha ofrecido cursos de postgrado sobre los siguientes temas: Tecnología Ambiental y Sostenibilidad en los Territorios Semiáridos, Fruticultura en el Semiárido, Educación Intercultural en el Pensamiento Decolonial, Procesamiento de Derivados en Frutas y Hortalizas y Procesamiento de Productos de Origen Animal.

Por considerarla de fundamental importancia, la institución mantuvo la política de calificación institucional de servidores a través de proyectos de Maestría y Doctorado Interinstitucionales (Minter/Dinter). Esta política está pautada en la planificación que busca la contratación de instituciones para la oferta de cursos en áreas específicas de interés institucional, de acuerdo con su Plan de Calificación Institucional (PQI). En esta misma perspectiva, el IF Sertão-PE ofrece cuotas de becas para servidores alejados para la realización de cursos de maestría y doctorado, a través del Programa Institucional de Calificación regulado (PIQ) por la Resolución No. 29 de 09 de septiembre de 2013.

\section{La pesquisa ambiental en el IF Sertão-PE}

El IF Sertão-PE todavía no tiene una política de sostenibilidad ambiental establecida y puesta en práctica. No existe la adhesión formalizada para participar en la Agenda Ambiental de la Administración Pública (A3P), aunque existe una propuesta de trabajo de la gestión construida para implementación en los siete campus/rectoría. En la institución se produce la separación de los residuos, por medio de la implantación de la Colecta Selectiva, sin embargo, aún no existe el destino dirigido a asociaciones y cooperativas de recolectores, aunque tal propuesta se encuentra en construcción por la gestión.

En lo que se refiere a contrataciones de empresas que aprecian la sostenibilidad, se observan los parámetros establecidos en el Decreto No. 7 746/2012, en lo que se refiere a potenciar la reducción de los impactos sobre recursos naturales, dar preferencia a materiales, tecnologías y materias primas de origen local y dar condición de mayor vida útil y menor costo de mantenimiento del bien y de la obra, entre otros aspectos. Sobre la constitución de la comisión gestora del Plan de Logística 
Sustentable (PLS) la institución ejecuta lo que dice el artículo No. 2, ítem IV) prácticas de racionalización; el elemento V) colecta selectiva, y el ítem XI) compra compartida (IF Sertão-PE, 2016).

En cuanto a la formalización y alcance del PLS aún no hay formalización de criterios, prácticas y acciones de logística sostenible. En cuanto a la indicación de donde se encuentra publicado el PLS y disponible en el sitio de la unidad, a pesar de cumplir los siguientes ítems del art. I) actualización del inventario de bienes y materiales del órgano o entidad e identificación de similares de menor impacto ambiental para sustitución; II) prácticas de sostenibilidad y de racionalización del uso de materiales y servicios; IV) acciones de divulgación, concientización y capacitación, los ítems I y II no se publican en el sitio institucional, estando sólo el ítem IV (IF Sertão-PE, 2016).

Además, los resultados obtenidos a partir de la implantación de algunas acciones aún no se publican semestralmente en el sitio del respectivo órgano, pero está siendo planeada su implementación a la mayor brevedad posible.

En este sentido, el IF Sertão-PE, por largos años, ignoró el debate sobre las cuestiones ambientales del punto de legislación, sin embargo, actualmente se ha buscado planificar, estructurar y buscar formas de implementación de diversos Programas Ambientales (A3P, PRS, PLS, etc.) indispensables para la sostenibilidad de una institución pública. De este modo, se han constituido comisiones responsables por la elaboración y posterior implantación de eventos y acciones de sensibilización, cursos de capacitación orientados a las demandas ambientales, aprobando el cumplimiento de los aspectos legales referentes a la gestión ambiental en la institución. Aunque algunos aspectos se han cumplido, éstos no poseen parámetros definidos, normativas internas instituidas, y se ejecutan en algunos sectores por algunos servidores, y en otros por algunos proyectos de investigación y/o extensión, de manera aislada y no como una política de sostenibilidad ambiental institucionalizada (IF Sertão-PE, 2016).

Muchos proyectos orientados a la cuestión ambiental han realizado prácticas ambientales sostenibles en el marco de dicha institución y son desarrollados por investigadores, los cuales además de ejecutar proyectos, han participado en asociaciones con instituciones locales que trabajan con cuestiones ambientales y han dado soporte, hecho consultorías, y realizado trabajos con diversas instituciones regionales, nacionales e internacionales.

\section{MATERIALES Y MÉTODOS}

El IF Sertão-PE está presente en las seis micro-regiones fisiográficas que constituyen el Sertão Pernambucano, según la Figura 1.

Figura 1. Localización del Sertão Pernambucano.

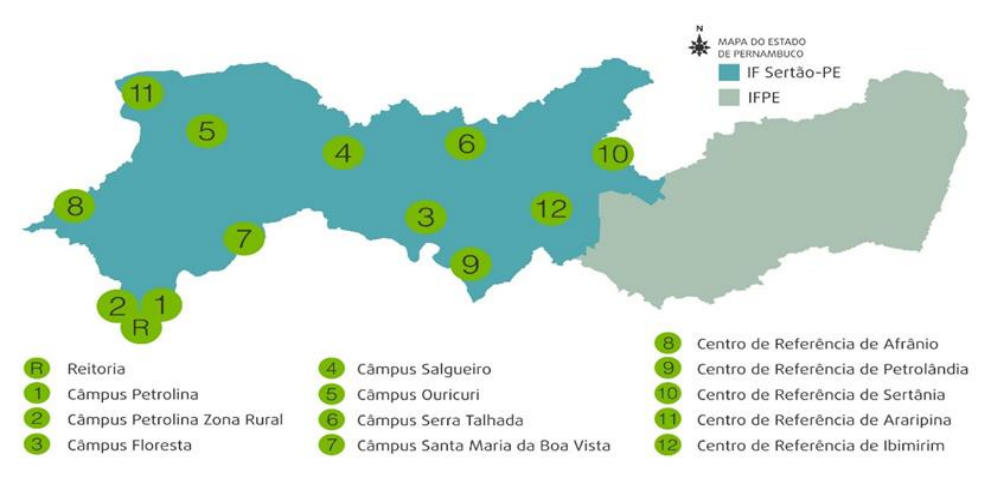

Fuente: IF Sertão-PE (2017b). 
Las acciones del Instituto Federal irradian hacia toda la región a partir de los siete campus ubicados en los municipios de Petrolina, Salgueiro, Floresta, Ouricuri, Santa María de Buena Vista y Sierra Talhada y de los centros de referencia en los municipios de Afrânio, Petrolândia y Sertânia.

El escenario geográfico donde está insertada enteramente la meso-región del Sertão Pernambucano es el semiárido, con condiciones climáticas desfavorables a las actividades relacionadas a la producción agrícola, y es la más extensa meso-región del estado. Su clima es cálido y seco, con temperaturas elevadas y régimen pluvial irregular (Iluvia escasa y mal distribuida durante el año). Tales condiciones climáticas se reflejan en la vegetación, los suelos y el régimen de los ríos, así como en la producción agrícola y en la movilidad de la población. Los suelos en su mayor extensión son rasos y con predominio de vegetación del tipo xerófilo, más adaptadas a climas secos y agua en pocas cantidades.

Con el fin de alcanzar sus objetivos estratégicos, el IF Sertão-PE planea, coordina, fomenta y acompaña las actividades de políticas de enseñanza, investigación aplicada, extensión e innovación tecnológica mediante articulación entre las pro-rectorías, direcciones sistémicas, departamentos y coordinaciones de forma indisociable. EI IF Sertão-PE superó la meta de atendimiento a la demanda del Programa de Becas de Iniciación Científica (PIBIC) prevista en el Programa de Desarrollo Institucional (PDI 20142018), atendiendo el $94 \%$ de la demanda de proyectos clasificados, mientras que la previsión era del 80\%. Las becas de la modalidad Programa de Becas de Iniciación Científica Júnior (PIBIC Jr.) también superó la meta del $80 \%$ prevista por el PDI 2014-2018, alcanzando el $84 \%$ de las propuestas clasificadas, según la Tabla 1.

Tabla 1. Número de becas ofrecidas, número de becas demandadas y atención a la demanda de becas de PIBIC y de PIBIC Jr., de 2012 a 2016, en el IF Sertão-PE.

\begin{tabular}{ccccccccc}
\hline Año & \multicolumn{9}{c}{ PIBIC } & \multicolumn{3}{c}{ PIBIC Jr. } \\
\cline { 2 - 9 } & IF & $\begin{array}{c}\text { Oferta } \\
\text { PIBIC/ } \\
\text { CNPq }\end{array}$ & $\begin{array}{c}\text { PIBIT/ } \\
\text { CNPq }\end{array}$ & $\begin{array}{c}\text { Demanda } \\
\text { (IF + CNPq) }\end{array}$ & $\begin{array}{c}\text { Tratamiento a } \\
\text { Demanda (\%) }\end{array}$ & Oferta & Demanda & $\begin{array}{c}\text { Tratamiento a } \\
\text { Demanda (\%) }\end{array}$ \\
\hline 2012 & 37 & - & - & 49 & 75,5 & 49 & 51 & 96,1 \\
2013 & 34 & - & - & 61 & 55,7 & 55 & 58 & 94,8 \\
2014 & 60 & - & - & 66 & 90,9 & 64 & 65 & 98,5 \\
2015 & 54 & 08 & 16 & 75 & 101 & 74 & 99 & 74,7 \\
2016 & 54 & 08 & 13 & 79 & 94,0 & 74 & 88 & 84,0 \\
\hline
\end{tabular}

Fuente: IF Sertão-PE (2017c).

Otra metodología propuesta por el PDI 2014-2018 era conceder la ayuda a la investigación al 100\% de los proyectos aprobados, por medio de becas APICPEX (Auxilio a Programas de Iniciación Científica y Proyectos de Extensión) se muestra en la Tabla 2.

Tabla 2. Número de proyectos de investigación e innovación tecnológica con APICPEX, 2016-2017.

\begin{tabular}{cccc}
\hline & $\begin{array}{c}\text { Proyectos } \\
\text { con bolsistas }\end{array}$ & $\begin{array}{c}\text { Proyectos con } \\
\text { APICPEX }\end{array}$ & $\begin{array}{c}\text { Relación } \\
\text { proyectos/APICPEX }\end{array}$ \\
\hline $33 / 2015$ & 150 & 86 & 0.57 \\
$16 / 2016-33 / 2016$ & 149 & 123 & 0.82 \\
\hline
\end{tabular}

Fuente: IF Sertão-PE (2017c, p. 50). 
Los avisos No. 16/2016 y No. 33/2016 seleccionaron 149 proyectos, de los cuales 123 propuestas solicitaron ayuda a los proyectos de investigación, o sea, el $82 \%$ de las propuestas aprobadas fueron contemplados con la ayuda (Tabla 3). La complejidad en la tramitación bancaria generó temores en algunos investigadores, justificando el $82 \%$. En relación al año anterior, hubo un aumento en la atención a la demanda del $57 \%$ al $82 \%$, resultado positivo que debe seguir creciendo hasta alcanzar la meta inicial del $100 \%$ de recibir la ayuda para todos los proyectos aprobados. Con la nueva forma de repaso de los recursos a los investigadores (vía tarjeta de investigación), que fue consolidada este año, esa meta será alcanzada.

Además, por medio del aviso No. 06/2016, se hizo pública la selección para preincubación e incubación de empresas de la ISA, cuatro propuestas fueron recibidas y cuatro propuestas fueron atendidas. En la Tabla 3 se presentan los indicadores de empresas pre incubadas e incubadas que se implementaron por vía pública desde 2013 a 2016.

Tabla 3. Número de empresas preincubadas e incubadas implementadas, vía aviso, de 2013 a 2016.

\begin{tabular}{lcccc}
\hline & Aviso 21/2013 & Aviso 12/2014 & Aviso 32/2015 & Aviso 6/2016 \\
\hline Empresas Preincubadas & 4 & 1 & 5 & 4 \\
Empresas Incubadas & 5 & 1 & 2 & 2 \\
\hline
\end{tabular}

Fuente: IF Sertão-PE (2017d, p.54).

En relación al incentivo a los servidores a calificarse, el IF SERTÃO-PE, en la Tabla 4 están relacionados los programas de postgrado, las instituciones promotoras, los niveles y las modalidades de oferta de cursos stricto sensu que poseen servidores del IF Sertão-PE en proceso de calificación. En 2016, se alcanzó la marca de 45 maestrandos y cinco doctorandos, totalizando la cantidad de 50 servidores cursando postgrado financiados por el IF Sertão-PE.

Tabla 4. Número de servidores atendidos por maestría y doctorado financiados por la institución, 2016.

\begin{tabular}{ccc}
\hline Curso & Universidad & $\begin{array}{c}\text { Número de } \\
\text { servidores }\end{array}$ \\
\hline Máster Profesional en Administración & UFBA & 15 \\
Máster Profesional en Educación & UFBA & 30 \\
Doctorado en Gestión & Évora - Portugal & 5 \\
Total & & $\mathbf{5 0}$ \\
\hline
\end{tabular}

Fuente: IF Sertão-PE (2017a, p. 12).

Entre tantas metodologías utilizadas para alcanzar las metas dentro de todos los sectores de la investigación, el IF Sertão-PE, a través de la Pro-Rectoría de Investigación, Innovación y Postgrado (PROPIP), promovió en 2016 una reunión con los líderes de grupos de investigación, catastrados en el Directorio del Consejo Nacional de Desarrollo Científico y Tecnológico (CNPq) durante la XI Jornada de Iniciación Científica y Extensión y contó con la participación de 23 líderes-investigadores.

Por último, se constata en el IF Sertão-PE, 46 Grupos de Investigación catastrados, con 468 investigadores involucrados y 208 líneas de investigaciones. La PROPIP alentó la actualización de los datos de los grupos de investigación y del currículo lattes, por medio de nota en el sitio electrónico y por e-mail institucional, enviado a los líderes, buscando también la participación en el Censo 2016 (CNPq). 
Por lo tanto, la PROPIP ha buscado incansablemente consolidar la investigación, propiciando la oportunidad para la participación de servidores y discentes de los diversos niveles y modalidades de enseñanza en proyectos de investigación.

\section{RESULTADOS Y DISCUSIÓN}

Entre las diversas metodologías empleadas por el IF Sertão-PE para consolidar la investigación dentro de la institución y derribar los muros institucionales para llegar a la sociedad, está la creación de estrategias de avance. En relación a las cuestiones ambientales varios proyectos e ideas se vienen consolidando en busca de la institucionalización de prácticas sustentables.

Se destaca la propuesta de creación e implantación del Núcleo de Investigación Geoambiental (NuPGeo) del IF Sertão-PE, por esta investigadora, inicialmente en el campus Petrolina y posteriormente, debe expandirse a los demás campus del instituto. En Brasil, la responsabilidad social de las instituciones públicas y privadas incluye la implantación de Centros de Estudio e Investigación en Medio ambiente para atender a la comunidad interna y externa.

La propuesta de creación del NuPGeo apunta a la formación de los actores sociales incorporando la cultura de la investigación aplicada en el área geográfica y ambiental, así mismo promociona entre los participantes el entendimiento del concepto de construcción de sociedad sostenible.

La idea de creación e implementación del Núcleo está fundamentada en la Política Nacional de Medio Ambiente del Brasil (Ley No. 6938, de 31 de agosto de 1981), que trata del papel de las instituciones públicas sobre la responsabilidad socioambiental.

EI NUPGEO, traerá al campus Petrolina del IF Sertão-PE una nueva dinámica de desarrollo del tríptico: enseñanza, investigación y extensión. Con el objetivo de construir una dinámica de motivación y capacitación a través de grupos de estudio e investigación de los participantes se espera construir una nueva visión a la investigación ambiental en esta institución, con miras al crecimiento de la investigación en el ámbito institucional y la valorización del crecimiento profesional de los participantes.

EI NUPGEO buscará alianzas nacionales e internacionales para el fortalecimiento de la investigación en el área geoambiental, concurrirá a las ediciones de fomento para la implantación de proyectos geoambientales, incentivará la participación de investigadores y estudiantes en grupos de estudio, monitoreos y proyectos de investigación. También implantará la Agenda A3P de la Administración Pública, para alcanzar los objetivos de la Agenda 2030 y la protección de la biodiversidad local. Además fortalecerá a través de la producción de libros, e-books, cartillas, informativos, boletines, la integración entre ciencia y tecnología en pro del conocimiento y de la sustentabilidad socioambiental y los valores de la solidaridad, la libertad, la diversidad, la justicia y la dignidad humana en favor de la cultura de la paz y la supervivencia de los ecosistemas y proporcionará la construcción de la ciudadanía planetaria, en la perspectiva crítica de conservar los recursos naturales para la actual y para las futuras generaciones.

Entre los proyectos aprobados en los avisos de este año, se tuvo el No. 19/2017 138 y de éste, 26 volcados para resolución de problemas ambientales en la región del semiárido. Los proyectos aprobados en el aviso No. 44/2017 y de los 21 proyectos, cinco son ambientales.

Siendo así, se percibe que los investigadores están empeñándose en desarrollar investigaciones que vengan a resolver diversos problemas vivenciados por la sociedad regional, aunque el número de 
proyectos ambientales aún resultan insuficientes si se tiene en cuenta la cantidad de investigadores en la institución y la cantidad de campus existentes.

Entre los proyectos y actividades desarrolladas dentro del IF Sertão-PE es posible destacar los siguientes:

- Taller de Medio ambiente y Sustentabilidad en los territorios semiáridos. Evento que ya forma parte del calendario de eventos de la institución, teniendo como objetivo reunir investigadores nacionales y regionales que discutan diversas temáticas ambientales, así como propongan soluciones y mitigaciones para las mismas. El evento ha reunido investigadores, estudiantes de todos los niveles y modalidades, público interno y externo al IF Sertão-PE, y ha obtenido diversos resultados, incluyendo publicaciones de libros y e-book con resultados de trabajos presentados durante el evento.

- Reunión de Líderes de Grupos de Investigación. Para trazar el levantamiento de actividades de investigación desarrolladas y en desarrollo por cada grupo de investigación, así como trazar directrices para cada día obtener investigaciones de excelencia que vengan a resolver la problemática social y ambiental.

- Encuentro de Investigación y Postgrado. Evento anual que se propone reunir a todos los coordinadores de cursos de postgrado del IF Sertão-PE, buscando la calificación por medio de la promoción de cursos, conferencias y talleres. El evento ha buscado consolidar la posgraduación dentro del Instituto Federal del Sertão Pernambucano.

- La Jornada de Iniciación Científica y Extensión (JINCE) y Jornada de Iniciación a la Docencia (JID). La primera ya está en su 12a edición y la segunda en su 1a edición. La jornada única ha promovido la integración de investigadores y becarios del IF Sertão-PE para la presentación de sus proyectos y esencialmente para el intercambio de experiencias en la enseñanza, la investigación, la extensión y la innovación. En este evento además de los trabajos orales, se presentan talleres, minicursos, muestras tecnológicas y didácticas, entre otras.

- La Semana Nacional de Ciencia y Tecnología. Evento que forma parte del calendario anual del Ministerio de Ciencia y Tecnología de Brasil, en el cual el IF Sertão-PE anualmente presenta en cada campus innumerables actividades de investigación e innovación tecnológica, propiciando la consolidación de la investigación en ámbito nacional.

- Taller de Innovación y Emprendedor. Evento realizado anualmente por el Instituto, ya se encuentra en su 4a edición, donde los dos últimos años se ha desarrollado en asociación con la Universidad Federal del Valle del São Francisco, con el objetivo de establecer la innovación y el espíritu empresarial en las instituciones de a nivel federal buscando favorecer a los emprendedores locales.

Además de las actividades que el Instituto desarrolla, a través de sus investigadores y extensionistas se logra la participación activa en eventos a través de asociaciones firmadas con universidades locales y regionales. Estos eventos se centran en cuestiones medioambientales locales. De este modo, varias son las acciones directas e indirectas del Instituto Federal para la consolidación de la investigación ambiental y sobre todo, aproximar la sociedad sertaneja de las acciones que buscan solucionar problemas externos de la comunidad. Entre ellas se destacan:

- Taller de Convivencia con el Semiárido que se encuentra en su 5a edición y busca consolidar propuestas de conservación del ecosistema caatinga.

- Seminario Verde Urbano Regional que tiene por objeto desarrollar acciones que impacten en cambios y transformaciones de los paisajes urbanos, consolidando áreas verdes y conservando los ambientes naturales.

- Congreso Brasileño de Educación Ambiental que está en su 3a edición y anualmente consolida la reunión de renombrados investigadores nacionales, potenciando la reflexión en torno a la 
educación ambiental en su ámbito legal y la relevancia de la práctica de acciones ambientales consolidadas.

- Las acciones del Día "C» de la Ciencia desarrolladas en asociación con las universidades estaduales y federales del Valle del São Francisco, Brasil, con el objetivo de la divulgación de los principales proyectos de enseñanza, investigación, extensión, innovación y medio ambiente, para acercar la sociedad de las investigaciones desarrolladas por tales instituciones.

\section{CONCLUSIONES}

El IF Sertão-PE utiliza varios mecanismos que permiten a la sociedad tener acceso a las acciones de la institución, como por ejemplo la divulgación en su portal electrónico y en sus medios sociales de procesos selectivos, cursos, proyectos, asociaciones, presupuesto, informes, entre otros, además del envío de informaciones para los medios de comunicación externo como radios, blogs y televisión. La institución también permite la inserción del ciudadano en la gestión pública de forma participativa, a través de la disponibilidad de un espacio destinado a la audiencia en el sitio, así como el e-SIC, además de la herramienta Hable con nosotros, también disponible en el sitio.

El IF Sertão-PE en los últimos años ha invertido en la capacitación y calificación de sus servidores, a través de la oferta de cursos de capacitación, sea por convenio con la Escuela de la Administración Federal (ESAF), con instructores internos o en instituciones externas.

Considerando que a partir de la conciencia comprometida con la responsabilidad ambiental, considerada actualmente uno de los mayores desafíos de la humanidad, el IF Sertão-PE inserto en ese contexto, debe buscar alternativas e involucrar a toda la comunidad a fin de mitigar los daños al Medio ambiente, adoptando procedimientos específicos para el uso racional de materias primas, insumos, energía, agua, aire y preocuparse por procesos productivos que causen menores daños a la comunidad, mediante la reducción de residuos sólidos, líquidos, basura, chatarra hacia productos de menor impacto ambiental.

Por lo tanto, es indispensable modificar la manera como se explotan los recursos naturales, y se pasa a vivir de la sustentabilidad, o perécela especie humana de forma brutal ahogada por sus propios residuos. Por lo tanto, es necesario inmediatamente que el Plan que se ha quedado sólo en el papel sea reevaluado, repensado y puesto en práctica a través de acciones que puedan mover y sensibilizar a toda la comunidad del IF Sertão-PE.

\section{REFERENCIAS BIBLIOGRÁFICAS}

Gobierno Federal de la República del Brasil. (1981). Política Nacional de Medio Ambiente. Ley No. 6 938 de 31 de agosto de 1981. http://www.Planalto.Gov.Br/Ccivil 03/Leis/L6938.Htm

Gobierno Federal de la República del Brasil. (2017). Agenda Ambiental de Administración Pública (A3p). Ministerio del Medio Ambiente. http://www.Mma.Gov.Br/Responsabilidade$\underline{\text { Socioambiental/A3p }}$

Instituto Federal del Sertão Pernambucano (2016). Informe Gestor Coordinación de Postgrado PROPIP. Relatoría de Gestión. http://www.Ifsertao-Pe.Edu.Br/Images/E-Sic/Relatorio-Gestao-2016.Pdf

Instituto Federal del Sertão Pernambucano (2017a). Programa de Desenvolvimiento Institucional (Pdi). https://www.Ifsertao-Pe.Edu.Br/Images/If Sertao-Pe/Documentos/Pdi\%202014-2018.Pdf

Instituto Federal del Sertão Pernambucano (2017b). Propuesta de Implementación del Núcleo de Pesquisa Geoambiental (NuPGeo). https://cutt.ly/RysdycD 
Instituto Federal Del Sertão Pernambucano (2017c). Pró-Rectoría de Pesquisa, Inovación y Posgrado (PROPIP) (2017). Informe de Gestión (2016-2017). https://www.ifsertaope.edu.br/images/Consup/2018/relatorio-gestor.pdf

Instituto Federal Del Sertão Pernambucano (2017d). Núcleo de Innovación Tecnológica (NIT). Informe de Gestión (2016-2017). https://www.ifsertao-pe.edu.br/images/Consup/2018/relatoriogestor.pdf

Pacheco, C. S. G. R. (2014). Ecodinámica del paisaje paleodunar del río São Francisco Medio / BA: en defensa de las fronteras atacadas. [Tesis de Maestría no publicada] Instituto de Tecnología de Pernambuco (ITEP)]. 